\title{
Positive predictive value of non-invasive prenatal screening for fetal chromosome disorders using cell-free DNA in maternal serum: independent clinical experience of a tertiary referral center
}

\author{
Whitney A. Neufeld-Kaiser ${ }^{1}$, Edith Y. Cheng ${ }^{2}$ and Yajuan J. Liu ${ }^{1 *}$
}

\begin{abstract}
Background: Non-invasive prenatal screening (NIPS) for fetal chromosome abnormalities using cell-free deoxyribonucleic acid (cfDNA) in maternal serum has significantly influenced prenatal diagnosis of fetal aneuploidies since becoming clinically available in the fall of 2011. High sensitivity and specificity have been reported in multiple publications, nearly all of which have been sponsored by the commercial performing laboratories. Once results are returned, positive and negative predictive values (PPVs, NPVs) are the performance metrics most relevant to clinical management. The purpose of this report is to present independent data on the PPVs of NIPS in actual clinical practice.

Methods: Charts were retrospectively reviewed for patients who had NIPS and were seen March 2012 to December 2013 in a tertiary academic referral center. NIPS results were compared to diagnostic genetic test results, fetal ultrasound results, and clinical phenotype/outcomes. The PPV was calculated using standard epidemiological methods. Correlation between screen results and both maternal age at delivery and gestational age at time of screening was assessed using Wilcoxon's rank sum test.

Results: Of 632 patients undergoing NIPS, $92 \%$ of tests were performed in one of the four major commercial laboratories offering testing. However, all four laboratories are represented in both the normal and abnormal results groups. There were 55 abnormal NIPS results. Forty-one of 55 abnormal NIPS results were concordant with abnormal fetal outcomes, 12 were discordant, and 2 were undetermined. The PPV for all conditions included in the screen was $77.4 \%(95 \% \mathrm{Cl}, 63.4$ - 87.3). Of 578 patients with normal NIPS results, normal pregnancy outcome was confirmed for 156 (27\%) patients. This incomplete follow-up of normal NIPS results does not affect PPV calculations, but it did preclude calculations of sensitivity, specificity, and NPV. Maternal age at delivery was significantly lower for patients with abnormal discordant results, compared to patients with abnormal concordant results $(P=0.034)$. Gestational age at time of screening was not associated with concordance of screen results $(P=0.722)$.

Conclusions: The experience of using NIPS in clinical practice confirms that abnormal results cannot be considered diagnostic. Pre-test counseling should emphasize this. Diagnostic genetic testing should always be offered following abnormal NIPS results.
\end{abstract}

Keywords: Cell-free DNA, NIPS, NIPT, Aneuploidy, Positive predictive value, Diagnostic genetic test

\footnotetext{
* Correspondence: yajuan@uw.edu

'Department of Pathology, Cytogenetics and Genomics Laboratory,

University of Washington School of Medicine, Box 357470, HSB H474B, 1959

NE Pacific Street, Seattle, WA 98195-7470, USA

Full list of author information is available at the end of the article
} 


\section{Background}

Cell-free deoxyribonucleic acid (cfDNA) refers to fragments of DNA circulating freely in the plasma. The fragments are most frequently $150-180$ base pairs in length $[1,2]$ and derive mostly from apoptotic cells [2]. During pregnancy, placental cytotrophoblastic cells are shed into maternal circulation and contribute to the cfDNA pool in the maternal bloodstream [3]. After 10 weeks gestation, an average of $10 \%$ of maternal cfDNA is placental in origin [4]. Non-invasive prenatal screening (NIPS) for fetal chromosome abnormalities is based on either massively parallel sequencing [5-11] or analysis of single nucleotide polymorphism (SNP) patterns $[12,13]$ from cfDNA in maternal serum. Very high sensitivity (98.6 - $100 \%)$ and specificity $(99.7-100 \%)$ have been reported in multiple clinical validation studies of NIPS for Down syndrome (DS) $[6,9,10,12,14]$. Somewhat lower sensitivities and specificities are seen when screening for trisomy 18 (T18), trisomy 13 (T13), and the sex chromosome aneuploidies (SCAs: 45,X; 47,XXX; 47,XXY; 47,XYY) [7-11, 13-17].

Although sensitivity and specificity are important performance metrics, positive predictive value (PPV) and negative predictive value (NPV) become more clinically relevant after results have returned. Although some publications sponsored by commercial laboratories performing NIPS have included PPV and NPV $[10,14,18,19]$, there is minimal independent data available on the performance of NIPS in actual clinical practice [20-23].

Here we present our first two years' experience with NIPS in a tertiary referral center. Performance was evaluated by calculating standard metrics such as PPV. Underlying biological causes for discordant results were determined where possible. Some discordant or unusual cases are described in detail. Benefits and limitations of using NIPS in clinical practice and recommendations for follow-up of abnormal results are discussed.

\section{Methods}

This study was approved by the Human Subjects Division (Application no. 47,683) and the Institutional Review Board (IRB) at the University of Washington Medical Center (UWMC). The requirement to obtain written consent and the requirement for Health Insurance Portability and Accountability Act (HIPAA) authorization were both waived by the IRB. Medical records were retrospectively reviewed for 632 consecutive patients who had NIPS and were seen between March 2012 and December 2013 in the Prenatal Genetics and Fetal Therapy (PGFT) Program, a tertiary referral center for prenatal genetic counseling and prenatal diagnosis located at UWMC. Patient age at delivery, gestational age at screening, indication for screening, and maternal serum screen results were extracted from outpatient clinic notes. Pregnancy outcomes were extracted from delivery summaries. Fetal ultrasound results were extracted from radiology records; diagnostic genetic test results were extracted from cytogenetics records; and NIPS results were extracted from laboratory records. Of the 632 patients undergoing NIPS, $92 \%$ of the tests were performed in one of the four major commercial laboratories offering testing during this timeframe.

Patients with normal NIPS results typically declined prenatal diagnostic genetic testing. If not already completed, maternal serum alpha-fetoprotein (AFP) and fetal anatomy ultrasound were recommended, and the patient was referred back to her primary obstetrical provider for routine care. Patients with abnormal NIPS results were offered high-resolution fetal ultrasound interpreted by either a UWMC perinatologist or a UWMC radiologist specializing in fetal anatomical imaging, genetic counseling, and prenatal diagnostic genetic testing via chorionic villus sampling (CVS) or amniocentesis. When the latter was declined, postnatal testing was recommended. Maternal chromosome analysis was added to our recommendations several months after we started to offer NIPS. For all patients, follow-up care recommendations were communicated verbally to the patient at the time of the NIPS results disclosure and/or genetic counseling consult, and in writing to the referring provider.

Normal NIPS results were defined as concordant when either diagnostic genetic test results matched the screen results or, for patients declining prenatal diagnostic genetic testing and delivering at UWMC, when normal pregnancy outcome was confirmed by review of delivery records. Newborn exams were performed by pediatricians. For patients with normal NIPS results who declined prenatal diagnostic genetic testing and delivered elsewhere, pregnancy outcomes were not confirmed. Abnormal NIPS results were defined as concordant when either diagnostic genetic test results matched the screen results or when multiple other clinical findings (such as fetal ultrasound abnormalities) corroborated the screen results. Nuchal translucency (NT) was defined as abnormal when the DS likelihood ratio was $\geq 2$ [24]. Patients who declined diagnostic genetic testing and were lost to follow-up with incomplete clinical information were not included in calculations of test performance. Diagnostic genetic test methods included interphase fluorescence in situ hybridization (IFISH), karyotyping, and cytogenomic microarray analysis (CMA). Diagnostic genetic testing was performed by the University of Washington Cytogenetics and Genomics Laboratory unless otherwise stated.

The $\mathrm{R}$ statistical software package (version $\mathrm{R}$ 2.12.0) [25] and Excel were used for all statistical analyses. For categorical data, frequencies or percentages with $95 \%$ confidence intervals were derived. For quantitative data, means with standard deviations, medians with minimum and maximum values, and frequencies were calculated. 
Wilcoxon's rank sum test was used to evaluate statistical significance between groups, and $P$-values of $\leq 0.05$ were considered statistically significant. The PPV was calculated with standard methods [26]. The reported performance of NIPS is remarkably similar across all test platforms in studies sponsored by the commercial laboratories $[6,9,10$, 12, 14, 19], so all NIPS results for this cohort were lumped, such that all performing commercial laboratories are represented therein.

\section{Results}

\section{Patient characteristics}

Of 632 patients, most were at increased risk for fetal aneuploidy as defined by American Congress of Obstetricians and Gynecologists guidelines [27]. Frequent indications for NIPS included maternal age $\geq 35$ at delivery, abnormal serum screen results, fetal ultrasound abnormalities, and family or personal history of a previous child with aneuploidy (Fig. 1). To determine whether concordance of NIPS results was correlated with maternal age at delivery or gestational age at screening, medians and their distributions were plotted and assessed for statistical significance (Fig. 2, Table 1). Maternal age at delivery was significantly older for patients with abnormal concordant results compared to patients with abnormal discordant results $(P=0.034)$ and to patients with normal results $(P=0.009)$. Gestational age at screening ranged from 9.9 to 33.6 weeks and was not significantly associated with concordance of results.

\section{PPV of NIPS in an independent clinical setting}

Figure 3 shows the breakdown of NIPS results for this patient cohort. Of 632 patients offered NIPS, no results were obtained for one patient, giving a test failure rate of $0.16 \%$. No redraws were needed in this cohort. Fifty-three of 631 (8\%) patients had abnormal NIPS results. This relatively high percentage reflects our high-risk patient population. Two patients had abnormal results for two chromosomes. One had abnormal results for DS and 47,XXX, and one had abnormal results for T18 and DS.

Among 55 abnormal NIPS results, 41 were concordant, 2 were undetermined and lost to follow-up, and 12 were discordant with diagnostic genetic testing and/or clinical phenotype (Fig. 3). Table 2 summarizes the clinical details of the 12 patients with discordant results. The overall PPV for all disorders included in the screen was 77.4 \% (95\% CI, 63.4 - 87.3). The highest PPV was for DS (90.3 \%, $95 \%$ CI 73.1 - 97.5). NIPS for T18 had the second highest PPV at $63.6 \%$ (95\% CI 31.6 - 87.6). The PPVs for the combined SCAs and for T13 were lowest, at $55.6 \%$ (95 \% CI 22.6 $84.6)$ and $50 \%$ (95 \% CI 2.7 - 97.3), respectively. If the 8 abnormal NIPS results that were compared to phenotype and clinical outcomes, instead of diagnostic genetic test results, are excluded, the overall PPV was $82.9 \%$ (95\% CI 68.6 - 91.9) in this cohort.

\section{Sex assessment}

NIPS results can also include fetal sex assessment, raising the possibility of normal results that are discordant for sex. We are aware of a single instance of normal NIPS results discordant for fetal sex during this timeframe. Patient 60 was a 26-year-old who presented at 12.4 weeks for routine aneuploidy screening. She had a personal history of congenital unilateral renal agenesis with contralateral damage secondary to reflux. She had undergone a renal transplant from a male donor three years prior. NIPS results were normal and consistent with a male fetus. During pre-test counseling and again when she was informed of the results, she was cautioned that the sex assessment may not be accurate in her case. Fetal anatomy ultrasound at 21.4 weeks showed female genitalia. She was counseled that the likely explanation for the gender discordance was that her cfDNA pool included Y chromosome DNA from the transplanted kidney. She declined amniocentesis for prenatal genetic

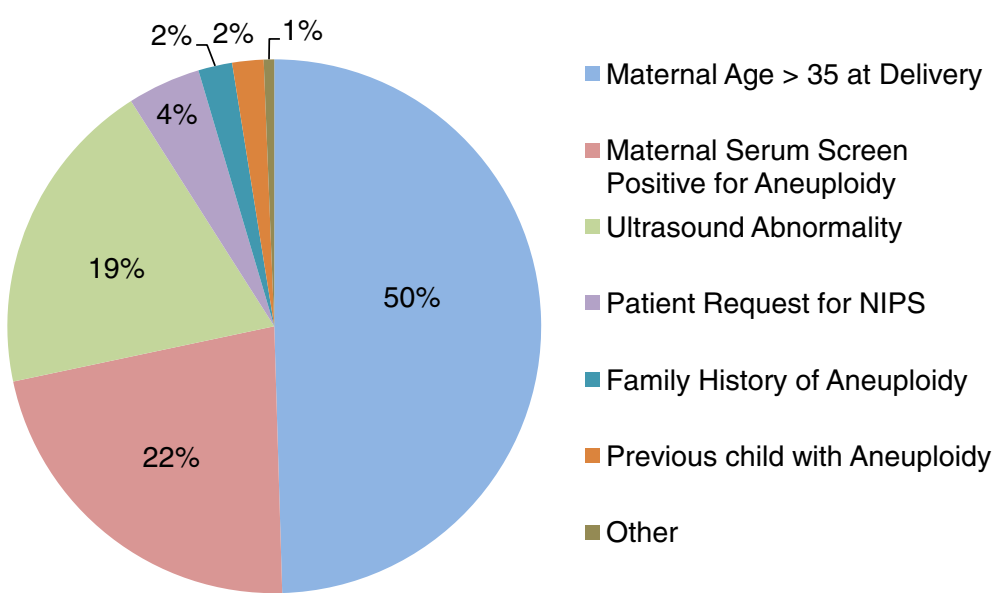

Fig. 1 Indications for NIPS. Abbreviations: NIPS, non-invasive prenatal screening 

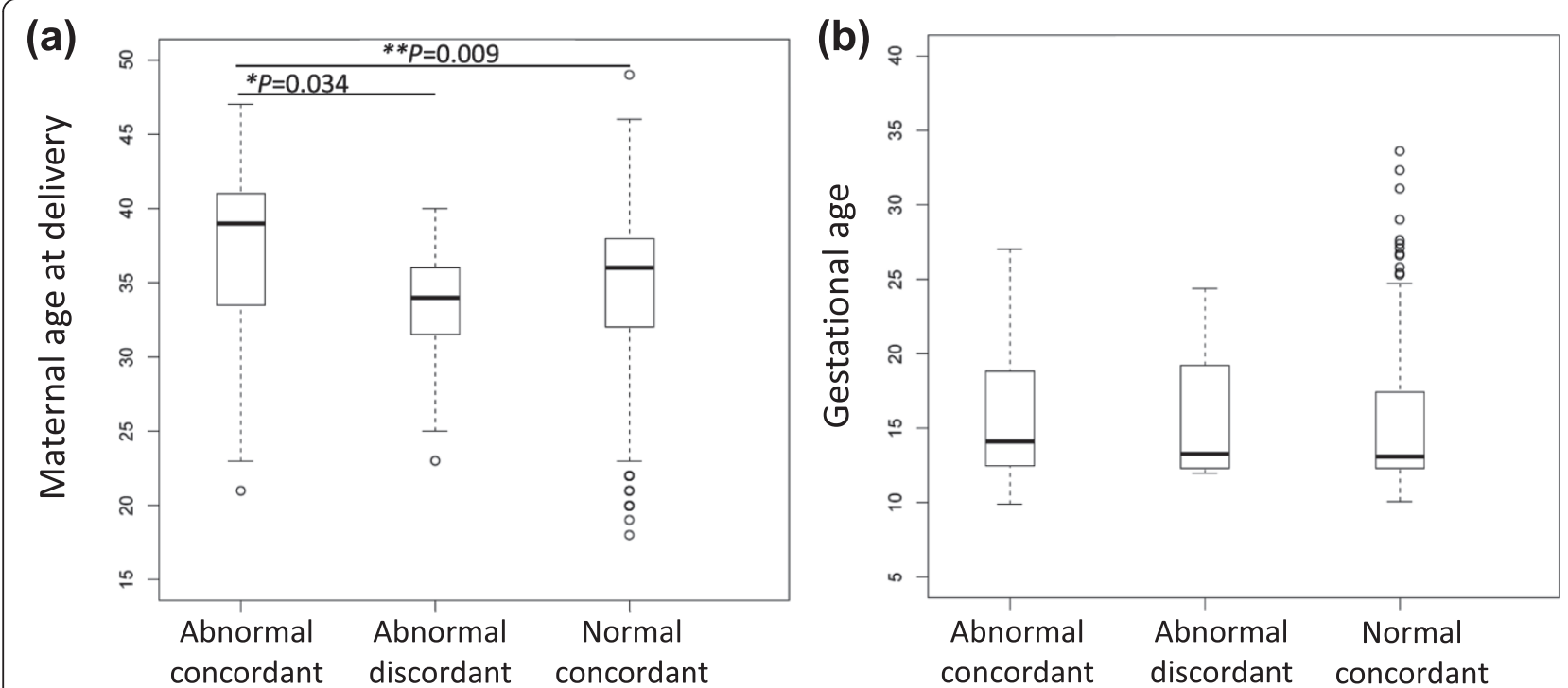

Fig. 2 Median and distribution of a maternal age at delivery and $\mathbf{b}$ gestational age at NIPS. P-values based on Wilcoxon's rank sum test $\left({ }^{*} P<0.05\right.$, $\left.{ }^{* *} P<0.01\right)$. Abbreviations: NIPS, non-invasive prenatal screening

diagnostic testing. The baby had normal appearing female external genitalia at birth. Karyotyping was not considered indicated and was not done prior to discharge.

\section{Follow-up after normal NIPS results}

Of 578 patients with normal NIPS results, 22 (4\%) had diagnostic genetic testing, the results of which were concordant in all cases for the chromosomes screened (Fig. 4). Diagnostic genetic testing was done postnatally on cord blood in 9 of the 22 cases secondary to congenital anomalies detected prenatally by ultrasound. In the other 13 cases, the patient chose to have prenatal testing by amniocentesis. Among these 13 patients, 3 opted for diagnostic testing after subsequent fetal anatomy ultrasound revealed anatomical anomalies; 5 stated an upfront preference for diagnostic genetic testing, but felt CVS was too risky, and used NIPS to assess fetal status at an early gestational age; 3 changed their minds during the prenatal genetic evaluation process and decided they preferred diagnostic testing; and the remaining 2 patients had unusual, unique, clinical circumstances that influenced their decision to pursue prenatal diagnostic genetic testing. Of patients with normal NIPS results who had no diagnostic genetic testing, 134 (23\%) had a normal pregnancy outcome confirmed by review of UWMC delivery records. Pregnancy outcome was not confirmed for the 422 (73\%) patients delivering elsewhere.

\section{Follow-up after abnormal NIPS results}

Fifty-three of 632 patients had abnormal NIPS results and were offered genetic counseling and follow-up testing. Figure 5 outlines patient decisions and outcomes after abnormal NIPS results. Follow-up fetal ultrasound results were normal in 12 of $53(23 \%)$ patients with abnormal NIPS results. NIPS results were discordant in 8 of 12 (67 \%) patients with normal follow-up fetal ultrasound results (Fig. 5, b and c). Fetal ultrasound anomalies were seen in the other 41 (77\%) patients with abnormal

Table 1 Maternal age at delivery and gestational age at time of NIPS, stratified by NIPS results

\begin{tabular}{|c|c|c|c|c|c|c|c|}
\hline & & & Median & Mean & SD & $P$-values ${ }^{\mathrm{a}}$ & \\
\hline \multirow[t]{3}{*}{ Maternal age (years) } & I. & Abnormal concordant & 39.00 & 36.97 & 6.13 & | vs. ||| & $0.009^{* *}$ \\
\hline & $\|$. & Abnormal discordant & 34.00 & 33.08 & 4.70 & | vs. || & $0.034^{*}$ \\
\hline & III. & Normal concordant & 36.00 & 34.79 & 5.16 & |I vs. III & 0.161 \\
\hline \multirow[t]{3}{*}{ Gestational age (weeks) } & ।. & Abnormal concordant & 13.10 & 14.94 & 3.74 & | vs. ||| & 0.156 \\
\hline & $\|$. & Abnormal discordant & 14.10 & 15.76 & 4.17 & | vs. || & 0.722 \\
\hline & III. & Normal concordant & 13.25 & 15.64 & 3.97 & || vs. III & 0.627 \\
\hline
\end{tabular}

${ }^{a} P$-values calculated using Wilcoxon rank sum test

${ }^{*} P<0.05$

**P $P<0.01$ 


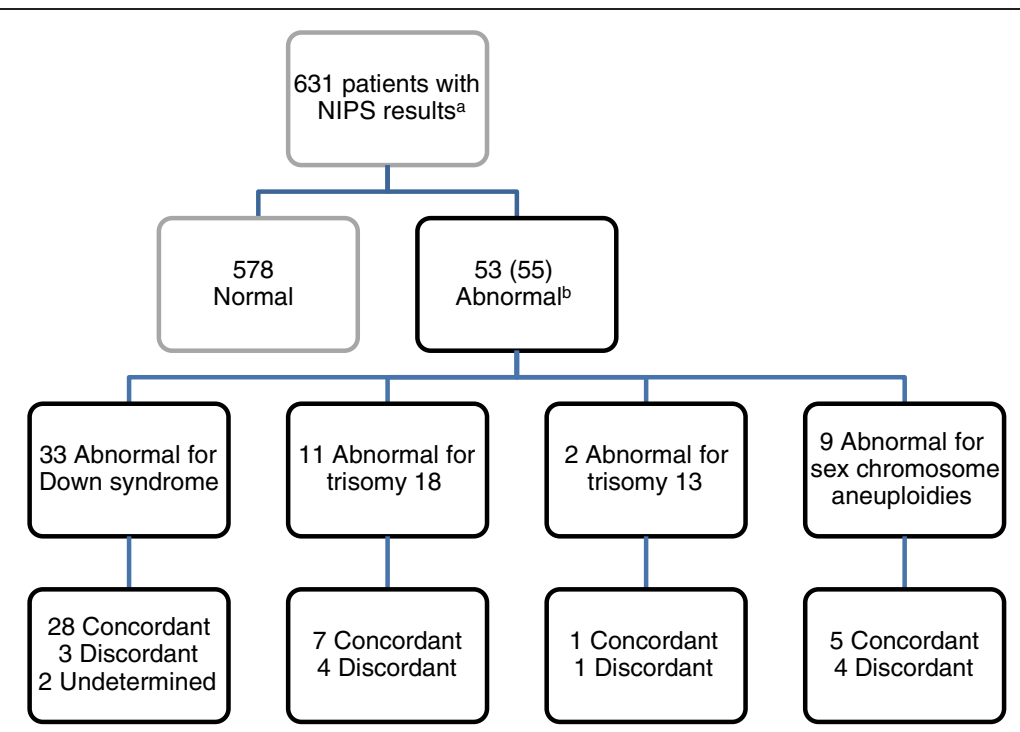

Fig. 3 NIPS results for this cohort. ${ }^{a}$ Sex chromosome aneuploidies were included in only 520 patients. ${ }^{\text {bTwo }}$ patients had abnormal results for two different chromosomes. Abbreviations: NIPS, non-invasive prenatal screening

NIPS results. NIPS results were discordant in 4 of 39 (10 \%) patients with abnormal follow-up fetal ultrasound results (Fig. 5 e, g, and h), excluding the 2 undetermined cases (Fig. 5j).

A genetic diagnosis was obtained in $45(85 \%)$ patients (Fig. 6). Twenty-two of 45 patients ( $49 \%$ ) chose prenatal diagnostic genetic testing (Fig. 5). Thirteen of these patients had concordant abnormal karyotype results and chose termination of pregnancy (TOP) (Fig. 5, a and d). The other 9, including 5 patients with discordant normal karyotype results (Fig. 5, b and e) and 4 patients with concordant abnormal karyotype results (Fig. 5, a and d), continued to term. Twenty-three of 45 patients (51\%) declined prenatal diagnostic testing, and postnatal testing was done instead (Fig. 5). Seven patients had an intrauterine fetal demise (IUFD) or neonatal demise (Fig. 5f). Seven patients opted for TOP after follow-up fetal anatomy ultrasound revealed anomalies consistent with the condition suggested by their NIPS results. Among these 14 patients, NIPS results were concordant with diagnostic genetic testing of fetal tissue in 14 of 15 instances. The single discordance occurred in a patient whose NIPS result was abnormal for both T18 and DS (Fig. 5g), as IFISH of fetal tissue showed two signals for chromosome 21 and three signals for chromosome 18. Nine patients continued to term. Neonatal peripheral blood karyotype results were concordant with NIPS results in 7 of these 9 cases (Fig. 5h).

For 8 patients who declined both prenatal and postnatal genetic testing, NIPS results were compared to clinical outcomes to evaluate concordance. Two of the 8 patients (one DS and one $45, \mathrm{X}$ ) had fetal ultrasonographic anomalies consistent with the condition suspected by NIPS, declined prenatal diagnostic genetic testing, had an IUFD, and declined postnatal genetic testing (Fig. 5i). These cases were considered concordant, because the probable diagnosis based on fetal anatomy ultrasound abnormalities matched the diagnosis suspected from NIPS results. Four of the 8 patients ( 3 T18, 1 DS) had normal follow-up fetal anatomy ultrasound results and declined prenatal diagnostic genetic testing (Fig. 5c). Newborn clinical exams performed by pediatricians were normal in all 4 cases, and postnatal genetic testing was not considered indicated. These cases were considered discordant because of the low likelihood that a newborn affected with T18 or DS would be phenotypically normal at delivery. Two patients with DS NIPS results had abnormal follow-up NT results, declined all further services, and opted for TOP (Fig. 5j).

\section{Discussion}

Publications regarding the performance of NIPS have mostly been sponsored by the commercial laboratories performing the test $[10,14,18,19,28]$. To our knowledge, this is the largest independent report of clinical experience using NIPS in a tertiary referral center in the United States. During the first two years of offering NIPS, we witnessed the powerful benefits of this technology. First, multiple studies have shown that NIPS has a very high NPV, including a recently published prospective multicenter study that found an NPV of $100 \%$ for DS [19]. Indeed, we are not aware of any normal discordant results among this patient cohort during this timeframe (Fig. 3). Normal NIPS results thus do greatly reduce the probability of a fetus affected with the conditions included in the screen. Whether NIPS is done as a primary screen 
Table 2 Details of discordant cases

\begin{tabular}{|c|c|c|c|c|}
\hline Patient & $\begin{array}{l}\text { NIPS } \\
\text { result }\end{array}$ & Other clinical information & Fetal diagnostic genetic test results & Maternal results \\
\hline 31 & $\begin{array}{l}\text { Abnormal } \\
\text { for DS }\end{array}$ & $\begin{array}{l}\text { US at } 19.3 \mathrm{w} \text { multiple anomalies consistent } \\
\text { with T18 }\end{array}$ & nuc ish (D21S259×2) on FFPE fetal tissue & Not offered \\
\hline 32 & $\begin{array}{l}\text { Abnormal } \\
\text { for DS }\end{array}$ & $\begin{array}{l}\text { US at } 12.3 \mathrm{w} \text { abnormal NT; US at } 20.7 \mathrm{w} \\
\text { normal; postpartum note "delivered a } \\
\text { male infant at } 36.6 \mathrm{w} . . . \text { birth weight } 6 \text { lbs } 10 \\
\text { oz...." }\end{array}$ & Declined & Not offered \\
\hline 33 & $\begin{array}{l}\text { Abnormal } \\
\text { for DS }\end{array}$ & $\begin{array}{l}\text { Paternal first cousin with DS; US at } 13.4 \mathrm{w} \\
\text { normal NT; US at } 16.6 \mathrm{w} \text { normal }\end{array}$ & $\begin{array}{l}46, X Y \text { in all } 15 \text { amniocyte clones; } \operatorname{arr}(1-22) \times 2,(X Y) \times 1 \\
\text { on amniocytes }\end{array}$ & $\begin{array}{l}46, X X \text { in all } 20 \text { peripheral } \\
\text { blood cells analyzed and } 30 \\
\text { additional cells screened for } 21\end{array}$ \\
\hline 41 & $\begin{array}{l}\text { Abnormal } \\
\text { for T18 }\end{array}$ & $\begin{array}{l}\text { AMA; FTS pos DS ( } 1 \text { in } 43) \text {; US at } 13.1 \mathrm{w} \\
\text { normal NT; US at } 17.6 \mathrm{w} \text { and } 19.7 \mathrm{w} \text { normal }\end{array}$ & Declined & Not offered \\
\hline 42 & $\begin{array}{l}\text { Abnormal } \\
\text { for T18 }\end{array}$ & AMA; US at $19.6 \mathrm{w}$ normal & $\begin{array}{l}\text { nuc ish(D18Z1×2)[200/200] on uncultured } \\
\text { amniocytes; } 46, X Y \text { in all } 52 \text { amniocyte clones }\end{array}$ & Not offered \\
\hline 43 & $\begin{array}{l}\text { Abnormal } \\
\text { for T18 }\end{array}$ & $\begin{array}{l}\text { AMA; US at } 12.7 \mathrm{~W} \text { normal NT; US at } 20.4 \mathrm{~W} \\
\text { normal }\end{array}$ & Declined & Not offered \\
\hline 44 & $\begin{array}{l}\text { Abnormal } \\
\text { for T18 }\end{array}$ & $\begin{array}{l}\text { US at } 19.0 \mathrm{w} \text { EIF; discharge exam: "Baby } \\
\text { does not have any physical exam findings } \\
\text { concerning for trisomy } 18 . "\end{array}$ & Declined & Not offered \\
\hline 46 & $\begin{array}{l}\text { Abnormal } \\
\text { for T13 }\end{array}$ & $\begin{array}{l}\text { AMA; US at } 13.7 \mathrm{~W} \text { normal NT; US at } \\
20.4 \mathrm{~W} \text { normal }\end{array}$ & $\begin{array}{l}\text { nuc ish(RB1X2)[50/50] on uncultured amniocytes; } \\
46, X X \text { in all } 15 \text { amniocyte clones }\end{array}$ & $\begin{array}{l}46, X X \text { in all } 20 \text { peripheral } \\
\text { blood cells analyzed and } 50 \\
\text { additional cells screened for } 13\end{array}$ \\
\hline 51 & $\begin{array}{l}\text { Abnormal } \\
\text { for } 45, X\end{array}$ & AMA; US at 19.3 normal & $\begin{array}{l}\text { nuc ish(DXZ1×2), (DYZ3X0)[191/200]; } 46, X X \text { in all } 21 \\
\text { amniocyte clones and } 100 \text { cells from mass culture }\end{array}$ & $\begin{array}{l}45, X[5] / 46, X X[45] \text { in peripheral } \\
\text { blood }\end{array}$ \\
\hline 52 & $\begin{array}{l}\text { Abnormal } \\
\text { for } 45, X\end{array}$ & $\begin{array}{l}\text { AMA; US at } 12.3 \mathrm{~W} \text { normal NT; US at } \\
20.3 \mathrm{w} \text { normal }\end{array}$ & $\begin{array}{l}\text { nuc ish(DXZ1x2)[220/225], (DYZ3X0)[225/225] on } \\
\text { uncultured amniocytes; } 46, X X \text { in all } 16 \text { amniocyte } \\
\text { clones analyzed and } 22 \text { additional clones screened } \\
\text { for } 45 X\end{array}$ & $\begin{array}{l}46, X X \text { in all } 20 \text { peripheral } \\
\text { blood cells analyzed and } 30 \\
\text { additional cells screened for } X\end{array}$ \\
\hline 55 & $\begin{array}{l}\text { Abnormal } \\
\text { for } 47, X X X\end{array}$ & $\begin{array}{l}\text { US at } 18.8 \mathrm{~W} \text { unilateral CPC and unilateral } \\
\text { renal pelviectasis }\end{array}$ & $\begin{array}{l}46, X X \text { in all } 20 \text { cells analyzed from peripheral } \\
\text { blood postnatally }\end{array}$ & $\begin{array}{l}46, X X \text { in all } 20 \text { peripheral } \\
\text { blood cells analyzed and } 30 \\
\text { additional cells screened for } X\end{array}$ \\
\hline 56 & $\begin{array}{l}\text { Abnormal } \\
\text { for } 47, X X X\end{array}$ & $\begin{array}{l}\text { Quad screen pos DS } 1 \text { in } 15 \text {, maternal } \\
\text { diabetes and renal failure, US at } 21.3 \mathrm{w} \\
\text { two-vessel cord }\end{array}$ & $\begin{array}{l}46, X X \text { in all } 20 \text { cells analyzed from peripheral } \\
\text { blood at } 7 \text { weeks of age }\end{array}$ & $\begin{array}{l}\text { Not done secondary to } \\
\text { chaotic family circumstances }\end{array}$ \\
\hline
\end{tabular}

Abbreviation: AMA advanced maternal age, CPC choroid plexus cyst, DS Down syndrome, EIF echogenic intracardiac focus, FFPE formalin-fixed, paraffin-embedded, FTS first trimester (maternal serum) screen, NT nuchal translucency, T18 trisomy 18, T13 trisomy 13, US ultrasound

${ }^{\mathrm{a}}$ Results were also abnormal for T18

at 10 weeks gestation or at 22 weeks after abnormal fetal ultrasound findings, normal results provide reassurance. Consequently, our rate of amniocentesis dropped significantly, a trend reported by others [20, 29-31]. In this series, $2 \%$ of patients with normal NIPS results chose prenatal diagnostic genetic testing, as compared to $42 \%$ of patients with abnormal results (Figs. 4 and 5).

Second, NIPS proved to be an invaluable tool for patients wanting to learn as much as possible about fetal health prior to delivery without incurring the miscarriage risk associated with CVS and amniocentesis. These patients commonly expressed that they would not consider TOP, regardless of the diagnosis. Even if NIPS results were abnormal, the family would benefit from being able to prepare for delivery with greater certainty about what lay ahead. In our cohort, this use of NIPS was especially obvious among patients confronted with abnormal findings on their mid-trimester fetal anatomy ultrasound, typically done at 18-22 weeks. Several of our patients in this situation voiced understanding that NIPS results are not diagnostic and still chose NIPS instead of diagnostic genetic testing (Fig. 1). Abnormal fetal ultrasound was the most frequent indication for NIPS (66 \%) among patients drawn after 20 weeks.

Our experience also confirmed the limitations of NIPS. First, only a handful of conditions are included in the NIPS panels, so normal results only lower the likelihood of the fetus being affected with those disorders. In this cohort, patient 58 was a 35 -year-old who presented at 13 weeks for routine screening. The NT measurement was markedly abnormal. The couple opted for NIPS, which returned with normal results. Fetal ultrasound at 16.3 weeks showed echogenic bowel, a two-vessel cord, unilateral clubfoot, unilateral renal agenesis, and an atrial septal defect. CMA and karyotyping of amniocytes showed mosaicism for trisomy 22, a condition not included in NIPS 


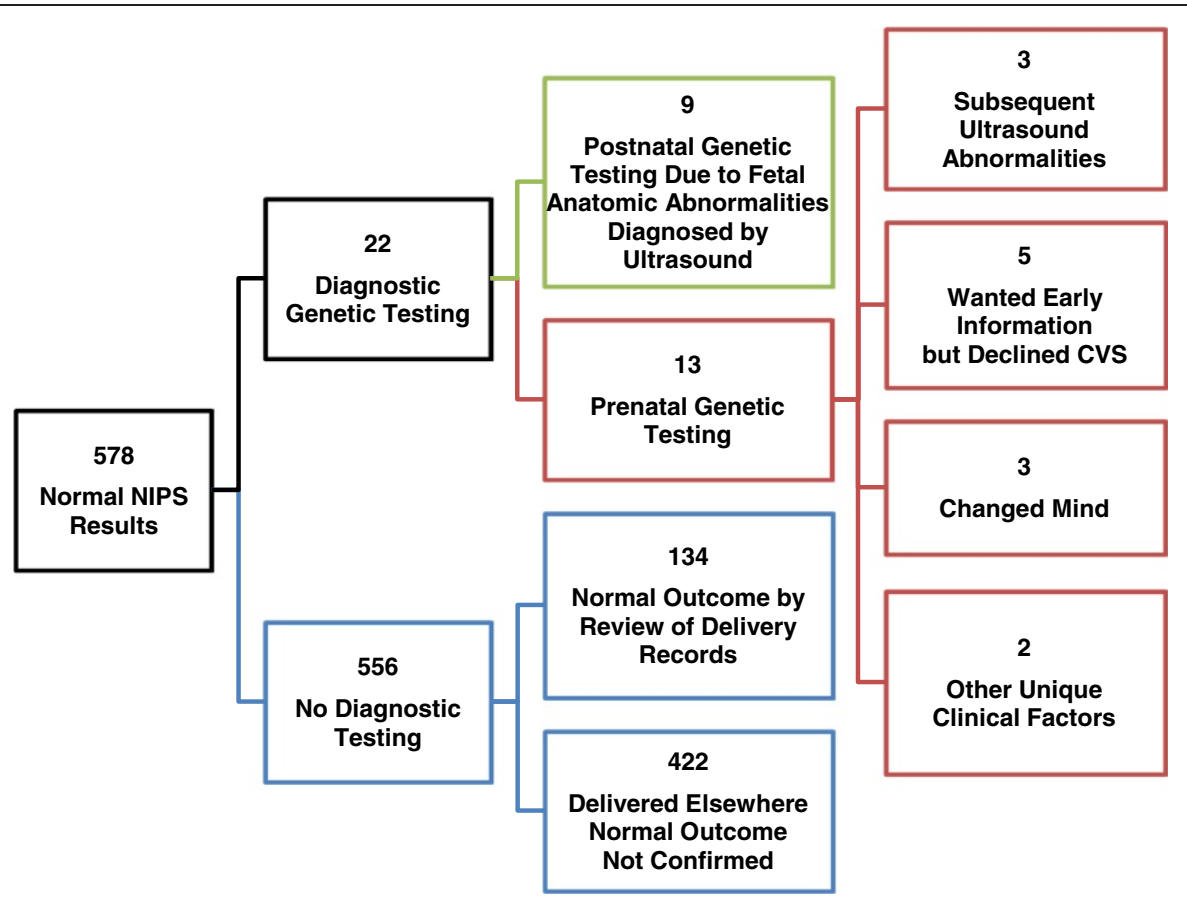

Fig. 4 Follow-up after normal NIPS results. Abbreviations: CVS, chorionic villus sampling; NIPS, non-invasive prenatal screening

at that time. Thus, patients with normal NIPS results should still be offered a fetal anatomy ultrasound to screen for congenital abnormalities.

Second, although normal discordant NIPS results are rare with such a high NPV, they do occur. Reports of "false negatives" have started to appear [32, 33], as the number of patients having NIPS has increased. Since data collection ended for this cohort, we have had one normal discordant result in a 34-year-old who presented at 13 weeks for routine screening. The NT measurement was enlarged. NIPS results were normal, but karyotyping of fetal tissue revealed $47, \mathrm{XY},+21$ in all cells.

Third, abnormal NIPS results confer a high risk, but not a diagnosis, of a fetal abnormality. The PPV was $77.4 \%$ (95 \% CI 63.4 - 87.3) in this cohort for all conditions included in the NIPS panels. We also observed that the PPVs differed in this cohort for each condition included in NIPS. Abnormal results for DS were the most frequent, and these results had the highest PPV. T18 was the next most frequent condition, and results had the second highest PPV. Abnormal results for the combined SCAs and T13 were the least frequent and had the lowest PPVs. This matches trends in the published literature $[10,14,18,28,34]$. There may be multiple reasons for this. T13 and T18 are less prevalent than DS, which would adversely impact the PPV. Confined placental mosaicism (CPM) for a trisomic cell line was observed more frequently at CVS for chromosomes 13 and 18 than for chromosome 21 [35, 36]. Postzygotic loss ("trisomy rescue") in a trophectoderm progenitor cell, leading to placental mosaicism for euploidy, was hypothesized to facilitate the intrauterine survival of T13 and T18 conceptuses [37]. The percentage of placental DNA in maternal circulation is generally lower when the fetus has T18, T13, or 45, $\mathrm{X}$ and higher when the fetus has DS $[4,28,38]$.

There are several causes of discordant results. Discordant results may be caused by statistical limitations of the analysis algorithms and/or uneven sequencing coverage secondary to guanine and cytosine (GC) content differences between chromosomes [39, 40]. PPV and NPV are influenced by the prevalence of the condition in the screened population. As expected in this cohort, maternal age at delivery was significantly younger among patients with abnormal discordant results, compared to patients with abnormal concordant results (Fig. 2a). Other studies have also shown a drop in PPV when women of all levels of risk are included, as compared to a solely high-risk population $[14,28]$. Normal discordant results have been reported to be more likely at an early gestational age because of a lower placental cfDNA fraction [6,7]. Gestational age at time of screening was not associated with concordance of results in our series (Fig. 2b), but we do not have data on body mass index and placental cfDNA fraction. Maternal mosaicism was detected in several instances of discordant NIPS results abnormal for an SCA [34, 41] and has also been reported for T18 [21]. Patient 51 (Table 2) was an example of this. Maternal chimera due to prior organ transplant was the likely cause of gender discordance for patient 60 (described above). CPM can cause discordant 


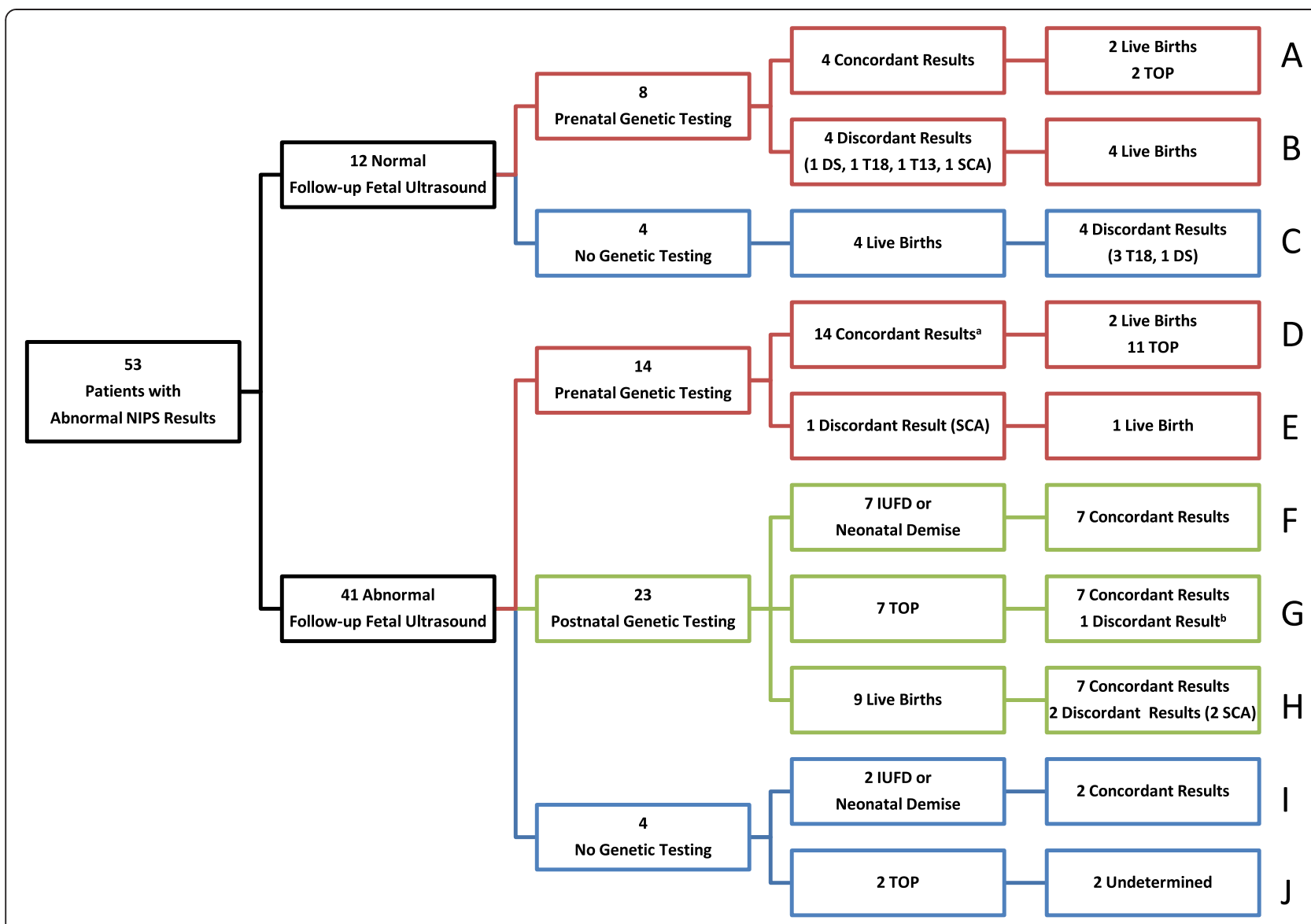

Fig. 5 Patient decisions after abnormal NIPS results and clinical outcomes. ${ }^{a}$ One patient had abnormal results for two different chromosomes (DS, XXX); both results were concordant. ${ }^{b}$ One patient had abnormal results for two different chromosomes; one result was concordant (T18) and one was discordant (DS). Abbreviations: DS, Down syndrome; IUFD, intrauterine fetal demise; NIPS, non-invasive prenatal screening; SCA, sex chromosome aneuploidy; T13, trisomy 13; T18, trisomy 18; TOP, termination of pregnancy

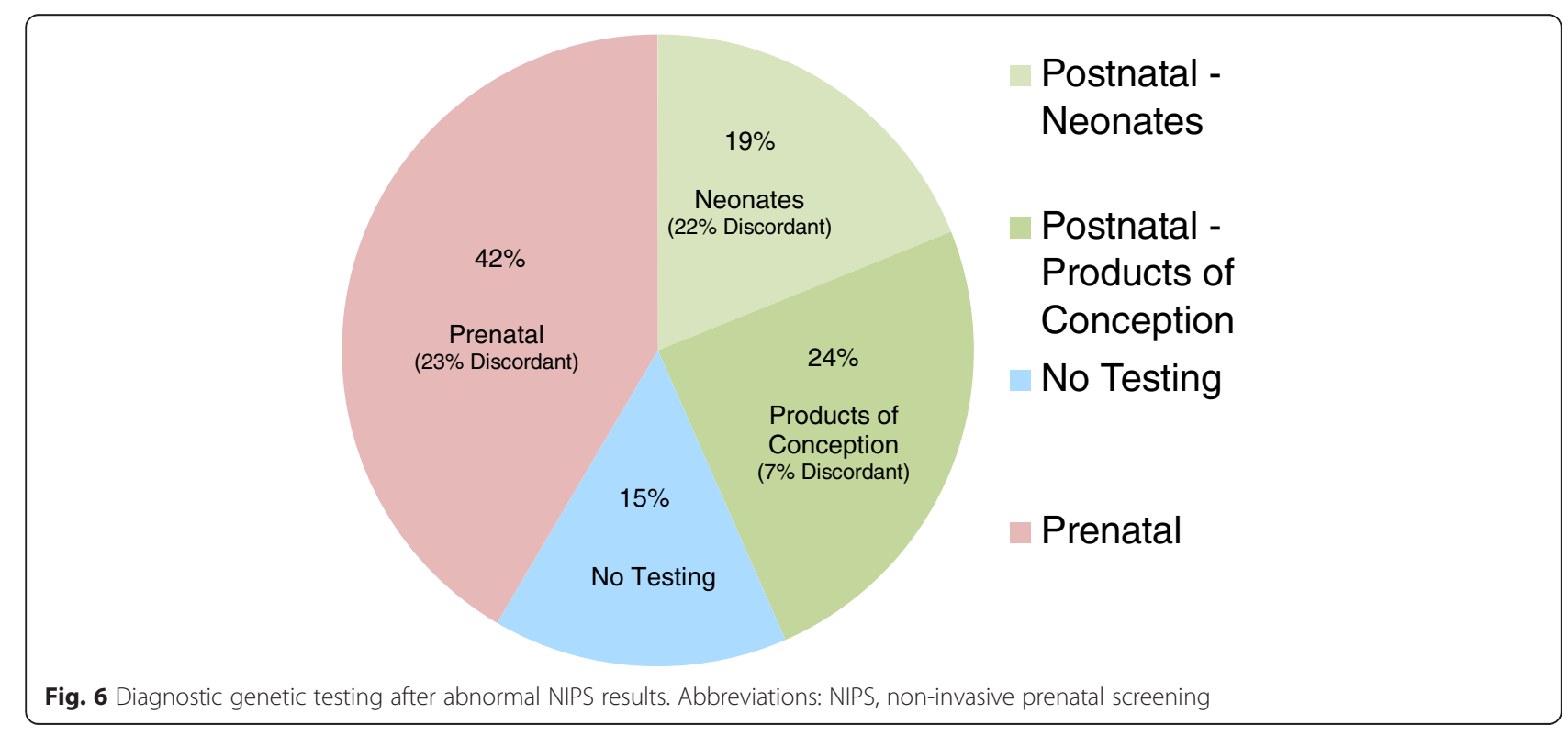


NIPS results, since the source of non-maternal cfDNA during gestation is the placental cytotrophoblastic cell line. Aneuploidy in the cytotrophoblastic cell line with a diploid fetus may cause an abnormal discordant NIPS result, whereas diploidy in the cytotrophoblastic cell line with a trisomic fetus may cause a normal discordant NIPS result $[32,42-44]$. There are several case reports of discordant NIPS results in pregnancies with proven CPM [33, 45-49]. Placental testing was not done in this cohort, because management is not influenced by results, but CPM may explain the discordant results in patient 46 (Table 2). Other biological reasons for discordant results include a vanishing twin with aneuploidy and maternal metastatic disease [50].

Apparent sex discordance can have a variety of causes, including inaccurate sex assessment on ultrasound, a cotwin demise, the statistical limitations of NIPS, and a fetus affected with one of the various disorders of sexual development. NIPS did not include the option of fetal sex assessment during the entire timeframe reported here, and not all patients chose to learn the predicted fetal sex from NIPS. Nevertheless, we are aware of only one instance of confirmed fetal sex discrepancy, in a patient who had a personal history of a renal transplant from a male donor.

Thus, it is imperative that providers make every effort to confirm NIPS results that are abnormal or appear discordant for fetal sex. Ideally, confirmation would be with diagnostic genetic testing done prior to making any irrevocable decisions about pregnancy management. Twenty-two families (42\%) in this cohort with abnormal NIPS results chose to have prenatal diagnostic genetic testing (Fig. 5a, b, d, e and Fig. 6). Nine patients in this group continued to term and took advantage of all opportunities to gain information about fetal health during their pregnancies. However, patients may decline prenatal diagnostic testing after an abnormal NIPS result, as did $31(58 \%)$ patients in this series. Patients incorporate other clinical information about fetal status into their decision-making. If serum screen results, NIPS results, and fetal anatomy ultrasound results all suggest the same diagnosis, the patient may not need further confirmation. Or a patient's concern about the suspected condition may not be sufficient to warrant the risk of the invasive procedures needed for prenatal diagnosis. And many patients pursue prenatal genetic screening to be better prepared for the birth, and would never incur the risk of a prenatal invasive procedure, regardless of NIPS results. Twenty-two patients in this cohort with abnormal NIPS results (41 \%) declined prenatal diagnostic genetic testing with the intention of continuing to term (Fig. 5c, f, h, and i).

Counseling patients about abnormal NIPS results is complex. The likelihood of an affected fetus depends on a priori risk and is influenced by what else is known clinically. For example, in this cohort, abnormal NIPS results were more likely to be discordant when fetal anatomy ultrasound was normal (67 \%) than when abnormalities were seen $(10 \%)$. Determining appropriate follow-up is also complicated. In some cases, doing a CVS to obtain diagnostic genetic test results may be the best approach, but it is problematic for several reasons. Since the non-maternal component of cfDNA during pregnancy is the placental cytotrophoblast, genetic testing via CVS is a repeat analysis of the same tissue type. If CVS reveals mosaicism, amniocentesis is recommended, and the pregnancy is subjected to two invasive procedures. Cytotrophoblasts are used for karyotyping in direct and short-term culture after CVS. Results from long-term CVS culture are generally based on analysis of the villi mesenchymal cores. Recommendations are to analyze both direct and long-term culture for the most accurate results $[35,36]$. Even so, CVS may not reveal $\mathrm{CPM}$, as only a small portion of the placenta is biopsied during the procedure. Select portions of the placenta may preferentially release cells into maternal circulation [48], and these may not be the same regions sampled by CVS. Patients should be counseled carefully about these limitations.

Multiple methods for diagnostic genetic testing are available. Karyotyping provides both numerical and structural information and distinguishes free trisomy from an unbalanced Robertsonian translocation. The latter can be inherited, with significantly increased recurrence risks when one parent is a carrier. However, chromosomal microdeletions have been added to NIPS panels, and these are typically undetectable with karyotyping. IFISH and CMA can detect both submicroscopic changes and aneuploidy, and these methods are more successful at obtaining a diagnosis from non-viable, frozen, or formalin-fixed, paraffin-embedded (FFPE) tissue [51]. But IFISH and CMA do not reveal structural rearrangements, such as unbalanced Robertsonian translocations causing DS.

Maternal karyotyping should be offered after abnormal NIPS results to rule out maternal mosaicism, especially when an SCA is suspected. Beyond possibly providing an explanation for abnormal NIPS results in a current pregnancy, patients with mosaicism are not good candidates for using NIPS in subsequent pregnancies.

For the 632 patients undergoing NIPS, $92 \%$ of the tests were performed in one of the four major commercial laboratories offering testing during this timeframe. Although the results mainly reflected one commercial lab doing NIPS, all four of those laboratories were represented in both the normal and the abnormal results groups of the cohort. In addition, the published performance of NIPS is remarkably similar across all test platforms in studies sponsored by the commercial laboratories $[6,9,10,12,14,19]$. 
Thus, the main emphases of this report, that the PPV of NIPS is less than $100 \%$ and abnormal results should be confirmed by diagnostic testing, apply to all NIPS platforms and methods currently in use.

A limitation of this report is that normal pregnancy outcome was not confirmed for $73 \%$ of patients with normal NIPS results. This precluded us from calculating sensitivity, specificity, or NPV for this cohort. However, the PPV of NIPS, the main focus of this report, is unaffected by outcomes of patients with normal NIPS results. Our center does not routinely collect outcome data for patients referred to us who do not deliver at our hospital, which is not unusual in a busy clinical practice. Based on our past experience with referral providers, we feel it likely that we would have been informed of any normal discordant result uncovered by the birth of a child affected with T13, T18, or DS. However, a normal discordant result could have remained undetected if a patient experienced a spontaneous pregnancy loss, and no postnatal genetic testing was done.

\section{Conclusions}

NIPS for fetal chromosome abnormalities using cfDNA in maternal serum is a powerful tool. It can be of enormous benefit to patients seeking information about fetal health. But with a PPV of less than $100 \%$, abnormal results cannot be considered diagnostic. Most ordering providers will not have time for a detailed discussion of sensitivity, specificity, PPV, and NPV when talking with patients about prenatal genetic screening options. At a minimum, providers should emphasize that 1) normal discordant results, though rare, do occur and 2) there are multiple possible explanations for an abnormal NIPS result. While an affected fetus is a common cause, it is not the only one. Management of an abnormal NIPS result is complex, and these patients should always be referred to a center offering genetic counseling, high-resolution fetal ultrasound, and diagnostic genetic testing.

\section{Consent}

This study was approved by the Human Subjects Division (Application no. 47,683) and the IRB at the UWMC. The requirement to obtain written consent and the requirement for HIPAA authorization were both waived by the IRB.

\footnotetext{
Abbreviations

AFP: alpha-fetoprotein; cfDNA: cell-free deoxyribonucleic acid; Cl: confidence interval; CMA: cytogenomic microarray analysis; CPM: confined placental mosaicism; CVS: chorionic villus sampling; DS: Down syndrome; FFPE: formalin-fixed, paraffin-embedded; GC: guanine and cytosine; HIPAA: Health Insurance Portability and Accountability Act; IFISH: interphase fluorescence in situ hybridization; IRB: Institutional Review Board; IUFD: intrauterine fetal demise; NIPS: non-invasive prenatal screening; NPV: negative predictive value; NT: nuchal translucency; PGFT: prenatal genetics and fetal therapy; PPV: positive predictive value; SCA: sex chromosome aneuploidy; SNP: single nucleotide polymorphism; T13: trisomy
}

13; T18: trisomy 18; TOP: termination of pregnancy; UWMC: University of Washington Medical Center.

\section{Competing interests}

The authors declare that they have no competing financial or non-financial interests.

\section{Authors' contributions}

WNK and YL had full access to all the data in the study and take responsibility for the integrity of the data and the accuracy of the data analysis. YL conceived of the study and performed the statistical analysis. WNK and YL participated in the study design and coordination, and drafted the manuscript. WNK, EC, and YL participated in acquisition, analysis, and interpretation of the data. All authors read and approved the final manuscript.

\section{Acknowledgement}

We acknowledge Xinxian Deng, Ph.D., Department of Pathology, University of Washington, Seattle for help with statistical analysis. No compensation was received by any person acknowledged here.

\section{Funding/support}

The Department of Pathology at the University of Washington, Seattle, Washington provides general funding and support for YL and WNK. The Department did not play a role in the design of the study; the collection, analysis, and interpretation of the data; in the writing of the manuscript; or the decision to submit the manuscript for publication.

\section{Authors' note}

Since the initial submission of this manuscript, it has come to our attention that three patients in our series have been included in other published reports. Patient 60 was also reported in Bianchi et al. Obstet Gynecol 2015;125(2):375. Patients 42 and 43 were also reported in Snyder et al. NEJM 2015; 372(17):1639-45.

\section{Author details}

'Department of Pathology, Cytogenetics and Genomics Laboratory, University of Washington School of Medicine, Box 357470, HSB H474B, 1959 NE Pacific Street, Seattle, WA 98195-7470, USA. ${ }^{2}$ Maternal Fetal Medicine and Medical Genetics, Departments of Obstetrics and Gyencology and Internal Medicine, University of Washington School of Medicine, Seattle, WA, USA.

Received: 12 December 2014 Accepted: 18 May 2015

Published online: 02 June 2015

\section{References}

1. Azmanov DN, Milachich TV, Zaharieva BM, Michailova Gl, Dimitrova VG, Karagiozova ZH, et al. Profile of chromosomal aberrations in different gestational age spontaneous abortions detected by comparative genomic hybridization. Eur J Obstet Gynecol Reprod Biol. 2007;131:127-31.

2. Suzuki N, Kamataki A, Yamaki J, Homma Y. Characterization of circulating DNA in healthy human plasma. Clin Chim Acta. 2008;387:55-8.

3. Faas BH, de Ligt J, Janssen I, Eggink AJ, Wijnberger LD, van Vugt JM, et al. Non-invasive prenatal diagnosis of fetal aneuploidies using massively parallel sequencing-by-ligation and evidence that cell-free fetal DNA in the maternal plasma originates from cytotrophoblastic cells. Expert Opin Biol Ther. 2012;12:S19-26.

4. Ashoor G, Poon L, Syngelaki A, Mosimann B, Nicolaides KH. Fetal fraction in maternal plasma cell-free DNA at 11-13 weeks' gestation: effect of maternal and fetal factors. Fetal Diagn Ther. 2012;31:237-43.

5. Diego-Alvarez D, Ramos-Corrales C, Garcia-Hoyos M, Bustamante-Aragones A, Cantalapiedra D, Diaz-Recasens J, et al. Double trisomy in spontaneous miscarriages: cytogenetic and molecular approach. Hum Reprod. 2006;21:958-66.

6. Palomaki GE, Kloza EM, Lambert-Messerlian GM, Haddow JE, Neveux LM, Ehrich $M$, et al. DNA sequencing of maternal plasma to detect Down syndrome: an international clinical validation study. Genet Med. 2011;13:913-20.

7. Palomaki GE, Deciu C, Kloza EM, Lambert-Messerlian GM, Haddow JE, Neveux $L M$, et al. DNA sequencing of maternal plasma reliably identifies trisomy 18 and trisomy 13 as well as Down syndrome: an international collaborative study. Genet Med. 2012;14:296-305.

8. Mazloom AR, Dzakula Z, Oeth P, Wang H, Jensen T, Tynan J, et al. Noninvasive prenatal detection of sex chromosomal aneuploidies by 
sequencing circulating cell-free DNA from maternal plasma. Prenat Diagn. 2013;33:591-7.

9. Bianchi DW, Platt LD, Goldberg JD, Abuhamad AZ, Sehnert AJ, Rava RP, et al. Genome-wide fetal aneuploidy detection by maternal plasma DNA sequencing. Obstet Gynecol. 2012;119:890-901.

10. Norton ME, Brar H, Weiss J, Karimi A, Laurent LC, Caughey AB, et al. Non-Invasive Chromosomal Evaluation (NICE) Study: results of a multicenter prospective cohort study for detection of fetal trisomy 21 and trisomy 18. Am J Obstet Gynecol. 2012;207:137. e131-138.

11. Ashoor G, Syngelaki A, Wang E, Struble C, Oliphant A, Song K, et al. Trisomy 13 detection in the first trimester of pregnancy using a chromosome-selective cell-free DNA analysis method. Ultrasound Obstet Gynecol. 2013;41:21-5.

12. Nicolaides KH, Syngelaki A, Gil M, Atanasova V, Markova D. Validation of targeted sequencing of single-nucleotide polymorphisms for non-invasive prenatal detection of aneuploidy of chromosomes 13, 18, 21, X, and $Y$. Prenat Diagn. 2013;33:575-9.

13. Samango-Sprouse C, Banjevic M, Ryan A, Sigurjonsson S, Zimmermann B, Hill M, et al. SNP-based non-invasive prenatal testing detects sex chromosome aneuploidies with high accuracy. Prenat Diagn. 2013;33:643-9.

14. Bianchi DW, Parker RL, Wentworth J, Madankumar R, Saffer C, Das AF, et al. DNA sequencing versus standard prenatal aneuploidy screening. N Engl J Med. 2014;370:799-808.

15. Rabinowitz M, Valenti E, Pettersen B, Sigurjonsson S, Hill M, Zimmermann B. Noninvasive aneuploidy detection by multiplexed amplification and sequencing of polymorphic Loci. Obstet Gynecol. 2014;123 Suppl 1:167S

16. Hall MP, Hill M, Zimmermann B, Sigurjonsson S, Westemeyer M, Saucier J, et al. Non-invasive prenatal detection of trisomy 13 using a single nucleotide polymorphism- and informatics-based approach. PLoS One. 2014;9:e96677.

17. Hooks J, Wolfberg AJ, Wang ET, Struble CA, Zahn J, Juneau K, et al. Non-invasive risk assessment of fetal sex chromosome aneuploidy through directed analysis and incorporation of fetal fraction. Prenat Diagn. 2014;34:496-9.

18. Futch T, Spinosa J, Bhatt S, de Feo E, Rava RP, Sehnert AJ. Initial clinica laboratory experience in noninvasive prenatal testing for fetal aneuploidy from maternal plasma DNA samples. Prenat Diagn. 2013;33:569-74.

19. Porreco RP, Garite TJ, Maurel K, Marusiak B, Ehrich M, van den Boom D, et al. Noninvasive prenatal screening for fetal trisomies 21, 18, 13 and the common sex chromosome aneuploidies from maternal blood using massively parallel genomic sequencing of DNA. Am J Obstet Gynecol. 2014;211:365. e361-365 e312

20. Beamon CJ, Hardisty EE, Harris SC, Vora NL. A single center's experience with noninvasive prenatal testing. Genet Med. 2014;16:681-7.

21. Song Y, Liu C, Qi H, Zhang Y, Bian X, Liu J. Noninvasive prenatal testing of fetal aneuploidies by massively parallel sequencing in a prospective Chinese population. Prenat Diagn. 2013;33:700-6.

22. Comas C, Echevarria M, Rodriguez MA, Prats $P$, Rodriguez I, Serra B. Initial experience with non-invasive prenatal testing of cell-free DNA for major chromosomal anomalies in a clinical setting. J Matern Fetal Neonatal Med. 2014;1-6.

23. Willems PJ, Dierickx H, Vandenakker E, Bekedam D, Segers N, Deboulle K, et al. The first 3,000 Non-Invasive Prenatal Tests (NIPT) with the Harmony test in Belgium and the Netherlands. Facts Views Vis Obgyn. 2014;6:7-12.

24. Herman A, Dreazen E, Herman AM, Batukan CE, Holzgreve W, Tercanli S. Bedside estimation of Down syndrome risk during first-trimester ultrasound screening. Ultrasound Obst Gynecol. 2002;20:468-75.

25. Team RDC. R: A language and environment for statistical computing. In. R Foundation for Statistical Computing: Vienna, Austria; 2008.

26. Fletcher RH, Fletcher SW. Clinical epidemiology: the essentials. 4th ed. Baltimore, MD: Lippincott Williams \& Wilkins; 2005.

27. American College of O, Gynecologists Committee on G. Obstet Gynecol. 2012;120:1532-4

28. Dar P, Curnow KJ, Gross SJ, Hall MP, Stosic M, Demko Z, et al. Clinical experience and follow-up with large scale single-nucleotide polymorphism-based noninvasive prenatal aneuploidy testing. Am J Obst Gynecol. 2014;211:527. e1-e17.

29. Larion S, Warsof SL, Romary L, Mlynarczyk M, Peleg D, Abuhamad AZ. Uptake of noninvasive prenatal testing at a large academic referral center. Am J Obst Gynecol. 2014;211:651. e1-e7.

30. Pettit KE, Hull AD, Korty L, Jones MC, Pretorius $\mathrm{DH}$. The utilization of circulating cell-free fetal DNA testing and decrease in invasive diagnostic procedures: an institutional experience. J Perinatol. 2014;34:750-3.

31. Louis-Jacques A, Burans C, Robinson S, Schofield E, Smulian J, Rochon M. Effect of commercial cell-free fetal DNA tests for aneuploidy screening on rates of invasive testing. Obstet Gynecol. 2014;123:1-67S.
32. Smith M, Lewis KM, Holmes A, Visootsak J. A case of false negative NIPT for Down syndrome-lessons learned. Case Reports Genetics. 2014;2014:823504

33. Wang Y, Zhu J, Chen Y, Lu S, Chen B, Zhao X, et al. Two cases of placental T21 mosaicism: challenging the detection limits of non-invasive prenatal testing. Prenat Diagn. 2013:33:1207-10.

34. Wang JC, Sahoo T, Schonberg S, Kopita KA, Ross L, Patek K, et al. Discordant noninvasive prenatal testing and cytogenetic results: a study of 109 consecutive cases. Genet Med. 2015;17:234-6.

35. Smith K, Lowther G, Maher E, Hourihan T, Wilkinson T, Wolstenholme J. The predictive value of findings of the common aneuploidies, trisomies 13, 18 and 21, and numerical sex chromosome abnormalities at CVS: experience from the ACC U.K. Collaborative Study. Association of Clinical Cytogeneticists Prenatal Diagnosis Working Party. Prenat Diagn. 1999;19:817-26.

36. Ledbetter DH, Zachary JM, Simpson JL, Golbus MS, Pergament E, Jackson L, et al. Cytogenetic results from the U.S. Collaborative Study on CVS. Prenat Diagn. 1992;12:317-45.

37. Kalousek DK, Barrett IJ, McGillivray BC. Placental mosaicism and intrauterine survival of trisomies 13 and 18. Am J Hum Genet. 1989:44:338-43.

38. Rava RP, Srinivasan A, Sehnert AJ, Bianchi DW. Circulating fetal cell-free DNA fractions differ in autosomal aneuploidies and monosomy X. Clin Chem. 2014;60:243-50.

39. Fan HC, Blumenfeld YJ, Chitkara U, Hudgins L, Quake SR. Noninvasive diagnosis of fetal aneuploidy by shotgun sequencing DNA from maternal blood. Proc Natl Acad Sci U S A. 2008;105:16266-71.

40. Chen EZ, Chiu RW, Sun H, Akolekar R, Chan KC, Leung TY, et al. Noninvasive prenatal diagnosis of fetal trisomy 18 and trisomy 13 by maternal plasma DNA sequencing. PLoS One. 2011;6:e21791.

41. Yao H, Zhang L, Zhang H, Jiang F, Hu H, Chen F, et al. Noninvasive prenatal genetic testing for fetal aneuploidy detects maternal trisomy $X$. Prenat Diagn. 2012;32:1114-6.

42. Grati FR, Malvestiti F, Ferreira JC, Bajaj K, Gaetani E, Agrati C, et al. Fetoplacental mosaicism: potential implications for false-positive and false-negative noninvasive prenatal screening results. Genet Med. 2014;16:620-4.

43. Srebniak MI, Diderich KE, Noomen P, Dijkman A, de Vries FA, van Opstal D. Abnormal non-invasive prenatal test results concordant with karyotype of cytotrophoblast but not reflecting abnormal fetal karyotype. Ultrasound Obstet Gynecol. 2014;44:109-11.

44. Pergament E, Cuckle H, Zimmermann B, Banjevic M, Sigurjonsson S, Ryan A, et al. Single-nucleotide polymorphism-based noninvasive prenatal screening in a high-risk and low-risk cohort. Obstet Gynecol. 2014;124:210-8.

45. Hall AL, Drendel HM, Verbrugge $J$, Reese AM, Schumacher KL, Griffith CB, et al. Positive cell-free fetal DNA testing for trisomy 13 reveals confined placental mosaicism. Genet Med. 2013;15:729-32.

46. Choi H, Lau TK, Jiang FM, Chan MK, Zhang HY, Lo PS, et al. Fetal aneuploidy screening by maternal plasma DNA sequencing: 'false positive' due to confined placental mosaicism. Prenat Diagn. 2013:33:198-200.

47. Pan M, Li FT, Li Y, Jiang FM, Li DZ, Lau TK, et al. Discordant results between fetal karyotyping and non-invasive prenatal testing by maternal plasma sequencing in a case of uniparental disomy 21 due to trisomic rescue. Prenat Diagn. 2013;33:598-601.

48. Mao J, Wang T, Wang BJ, Liu YH, Li H, Zhang J, et al. Confined placental origin of the circulating cell free fetal DNA revealed by a discordant non-invasive prenatal test result in a trisomy 18 pregnancy. Clin Chim Acta. 2014;433:190-3.

49. Gao $Y$, Stejskal D, Jiang F, Wang W. False-negative trisomy 18 non-invasive prenatal test result due to $48, X X X,+18$ placental mosaicism. Ultrasound Obstet Gynecol. 2014;43:477-8.

50. Osborne CM, Hardisty E, Devers P, Kaiser-Rogers K, Hayden MA, Goodnight W et al. Discordant noninvasive prenatal testing results in a patient subsequently diagnosed with metastatic disease. Prenat Diagn. 2013;33:609-11.

51. Reddy UM, Page GP, Saade GR, Silver RM, Thorsten VR, Parker CB, et al. Karyotype versus microarray testing for genetic abnormalities after stillbirth. N Engl J Med. 2012;367:2185-93. 